\title{
Procesos de subjetivación y lenguaje inclusivo*
}

\author{
Processes of subjectivization and inclusive language
}

\section{María Marta García Negroni** , Beatriz Hall ${ }^{* * *}$}

\section{RESUMEN}

En este trabajo, sin afán alguno de establecer juicios que auto-

Palabras clave: ricen o nieguen la pertinencia de los usos del lenguaje inclusivo, proponemos un análisis lingüístico-discursivo de las resoluciones emitidas por cuatro universidades argentinas que han aceptado el uso del lenguaje inclusivo en alguna de sus formas y en distintos tipos de producciones académicas escritas y orales. Nuestro objetivo es mostrar que el sentido de los discursos no es de naturaleza veritativa, ni depende de las elecciones vocausas de la enunciación, dialogismo, lenguaje inclusivo, marcos de discurso, subjetividad. luntarias e intencionales de los sujetos; antes bien, los efectos de sentido se construyen dialógica y argumentativamente. A la luz del enfoque dialógico de la argumentación y la polifonía, proponemos así que las mencionadas resoluciones exponen sistemáticamente una imagen de la enunciación como causada por (semi)marcos de discurso dialógicos que deben recuperarse y que remiten a prácticas discursivas que toman el uso mismo del lenguaje como objeto y su correlato en las prácticas sociales. Al mismo tiempo, mostramos cómo, en esas respuestas dialógicas, quedan materializados posicionamientos subjetivos críticos que, aunque diversos, devuelven a los sujetos la ilusión de ser dueños de su decir.

\footnotetext{
* El presente trabajo se inscribe en el proyecto de investigación PICT 2943/17 “El enfoque dialógico de la argumentación y de la polifonía. Un modelo semántico-pragmático para el análisis de la subjetividad y de la alteridad en distintos géneros discursivos", subvencionado por la Agencia Nacional de Promoción Científica y Tecnológica, Argentina. ** Argentina. Doctora en Ciencias del Lenguaje. Profesora asociada en la Universidad de San Andrés/Conicet, Buenos Aires, Argentina. mamagn@gmail.com

*** Argentina. Doctora en Lingüística. Profesora adjunta en la Universidad de Buenos Aires. Profesora asociada en la Universidad Nacional de Lomas de Zamora, Buenos Aires/Lomas de Zamora, Argentina. hallbeatriz@gmail.com
} 


\begin{abstract}
In this paper, without wanting to deny the use of inclusive language's relevance, we propose a linguistic-discursive analysis of the resolutions issued by four Argentine universities that have accepted inclusive language in some of its forms and different types of written and oral academic productions. We aim to show that the discourse's meaning is not of a veritative nature or depends on the subjects' voluntary and intentional choices. Rather, the effects of meaning construct dialogically and argumentatively. In light of the dialogical approach to argumentation and polyphony, we propose that the resolutions mentioned above systematically expose an enunciation image caused by (semi-) dialogical discourse. Frameworks that must be recovered and refer to discursive practices that take the very use of language as an object and its correlation in social practices. At the same time, we show how in these dialogical responses, critical subjective positions remain materialized that, although diverse, give the subjects the illusion of being masters of their saying.
\end{abstract}

Keywords: causes of enunciation, dialogism, inclusive language, discourse frameworks, subjectivity. 


\section{Introducción}

Mucho se ha dicho acerca de la no coincidencia entre el género gramatical y la identidad de las personas. En términos generales, este planteo ha dividido aguas entre quienes se han referido al tema desde el punto de vista académico-científico de la lengua y quienes incluyen en esta discusión aspectos que exceden lo lingüístico.

Las perspectivas basadas en las posibilidades del sistema lingüístico se presentan como cargadas de un mayor grado de cientificismo y, por lo tanto, de certeza cuando se acepta o se rechaza el uso de diferentes terminaciones para nombres, pronombres, artículos, cuantificadores y adjetivos relacionados con seres humanos. Así, por ejemplo, la RAE defiende el uso del masculino genérico como el mecanismo inclusivo que tiene la lengua para aludir a colectivos formados por hombres y mujeres, no acepta el uso de la x y de la @ -por la dificultad de la pronunciación que ambas opciones plantean-, desestima las formas abstractas y los desdoblamientos -por innecesarios o artificiosos- y afirma que el uso de la $e$ es ajeno al sistema morfológico del español como marca de género. Las expresiones como todes o chiques constituyen para la RAE formas no válidas para referir a identidades.

Sin embargo, el valor normativo de las instituciones que regulan los usos de la lengua para informar a los usuarios acerca de cuáles de esos usos son aceptados y cuáles no lo son se ha visto fuertemente cuestionado en estos últimos años -sobre todo en lo referido al lenguaje inclusivo-. Así, por ejemplo, desde una perspectiva radicalmente diferente, Salerno (2019) señala que el "gramacentrismo" (es el término que utiliza la autora) desoye el carácter discursivo del fenómeno. Es más, adhiere al término de lenguaje "incisivo", en lugar de inclusivo, para referir específicamente a un lenguaje que no discute una norma gramatical, sino una norma institucional y social. "Lo discutido es el uso del género gramatical masculino para referirse a una identidad de género masculina, femenina, no binaria, trans, lesbiana, intersex, etc., afirma Salerno (2019, p. 112). La $e$, la $x$ o la @ serían entonces incisivas, porque cuestionarían la lengua hegemónica y señalarían el rechazo a la interpretación dicotómica que equipara identidad de género y sexo biológico.

Entre otros autores, Kalinowski (en Sarlo y Kalinowski, 2019) -que ve en el uso del masculino genérico el correlato gramatical de un reor- 
denamiento social patriarcal que remite a la discriminación a la que las mujeres se han visto sometidas durante siglos-, afirma que "el objetivo del inclusivo no es volverse gramática" (p. 33). Se trataría en todo caso de un fenómeno político, discursivo y retórico de lucha por la igualdad en la sociedad que busca crear consciencia. Por ello, para Kalinowski, no estamos ante un hecho de lengua y la aparición de los usos inclusivos no constituye un cambio lingüístico ya que, según afirma en un artículo periodístico publicado en La Nación el 18 de febrero de 2020, estos se dan inconscientemente y los hablantes no los deciden. Se trata solo de una intervención del discurso público que expresa un posicionamiento político o ideológico.

Por el contrario, para Martínez (2019), estaríamos presenciando un cambio que apunta al sistema lingüístico. Según la autora, el sistema binario de género del español, que resultó eficiente durante siglos, está empezando "a no 'encajar' al menos para algunas personas que desean manifestar que en una porción de ese paradigma se esconde una manipulación" (p. 10). Las necesidades comunicativas actuales estarían llevando a que el fonema $e$ adquiera estatus de morfema y eso "sería el germen de la introducción de un paradigma de género humano" (p. 11). En ese nuevo paradigma la sustancia semántica "género humano" estaría categorizada como masculino, femenino y otro(s), este último entendido como el que corresponde a lo que no es masculino o femenino "porque, o son ambos a la vez o bien se trata de una opción diferente":

\begin{tabular}{cc}
\hline MASCULINO & {$[\mathrm{O}(\mathrm{S})]$} \\
\hline FEMENINO & {$[\mathrm{A}(\mathrm{S})]$} \\
\hline OTRO(S) & {$[\mathrm{O}(\mathrm{S})] /[\mathrm{E}(\mathrm{S})]$} \\
\hline
\end{tabular}

Fuente: Martínez (2019, p. 11).

Sea como sea, la utilización de la x(todxs), de la@(niñ@s), de los desdoblamientos (niños y niñas), el recurso de las formas abstractas (la juventud, la ciudadanía) y el empleo de la $e$ (chiques) constituyen, sin duda, distintas propuestas que, cada vez con mayor fuerza, buscan evitar el sexismo y objetar el binarismo en el lenguaje. Claramente se trata de una lucha simbólica y política que se produce en el campo discursivo para poner fin a la invisibilización de la mujer, así como de otras identidades de género que han sido tradicionalmente silenciadas. 
En este trabajo, nuestro objetivo es aportar a la discusión acerca del lenguaje inclusivo mediante el análisis de su utilización como respuesta dialógica a marcos de discurso referidos a prácticas discursivas actuales y como la plasmación de posicionamientos enunciativos críticos que devuelven a los sujetos la ilusión de ser dueños de su decir (Authier-Revuz, 1984). Para ello, y a la luz del enfoque dialógico de la argumentación y la polifonía (García Negroni, 2016; 2018a; 2018b; 2019a; García Negroni y Libenson, 2020; García Negroni y Libenson, en prensa), analizamos un corpus de textos compuesto por las resoluciones emitidas por cuatro dependencias universitarias argentinas que han aceptado el uso del lenguaje inclusivo en alguna de sus formas y en distintos tipos de producciones académicas escritas y orales (grado, posgrado, administrativo, técnico, etc.). Se trata de las resoluciones de la Universidad Nacional de San Martín (de aquí en más UNSAM), de marzo 2019; de la Universidad Nacional de Entre Ríos (de aquí en más UNER), de agosto de 2019; y de las facultades de Ciencias Sociales (de aquí en más FSoc) y de Filosofía y Letras (de aquí en más FFyL) de la Universidad de Buenos Aires, de julio y diciembre de 2019, respectivamente ${ }^{1}$.

En lo que sigue, presentamos, en primer lugar, las herramientas teórico-metodológicas del enfoque dialógico de la argumentación y la polifonía (de aquí en más EDAP), en el que se sustenta nuestra investigación. Luego, nos abocamos al análisis y comparación de las distintas resoluciones del corpus con el objetivo de poner de relieve no solo sus similitudes y sus diferencias, sino sobre todo cómo en cada una de ellas, en tanto respuestas dialógicas, quedan plasmados posicionamientos subjetivos que, aunque diversos, materializan la representación de un sujeto que busca controlar el decir. Por último, en la última sección introducimos algunas reflexiones finales.

1 Hasta el momento, las universidades que se han expedido sobre el uso del lenguaje inclusivo en sus dependencias son las siguientes: Universidad Nacional de Río Negro (2017), Facultad de Humanidades y Ciencias Sociales de la Universidad Nacional de la Patagonia San Juan Bosco (2019), Universidad Nacional de Mar del Plata (2019), Universidad Nacional de Córdoba (2019), Universidad Nacional de Rosario (2019). 


\section{El enfoque dialógico de la argumentación y la polifonía y las causas dialógicas de la enunciación}

En la senda trazada por las teorías del dialogismo (Bajtín, 1982), de la polifonía (Ducrot, 1984) y de la argumentación en la lengua (Anscombre, 1995; Anscombre \& Ducrot, 1983; Carel y Ducrot, 2005; Ducrot, 2004), el EDAP caracteriza los discursos que evoca toda enunciación como intrínsecamente argumentativos y los analiza como respuesta siempre dialógica frente a discursos previos o potenciales. En otras palabras, el análisis semántico que propone el EDAP mantiene el principio ducrotiano según el cual el sentido de nuestras palabras, expresiones o enunciados no está constituido por las cosas, los hechos o las propiedades que denotan, ni por los pensamientos o las creencias que sugieren, sino por los encadenamientos o discursos argumentativos a los que esas palabras, expresiones o enunciados aluden. Esos discursos argumentativos pueden ser normativos o transgresivos (Carel y Ducrot, 2005). En el primer caso, el encadenamiento aparece articulado por conectores conclusivos del tipo de por lo tanto, si... entonces, porque, en consecuencia, así que, etc. (realizaciones de un conector abstracto POR LO TANTO, abreviado como PLT); en el segundo, por conectores concesivos del tipo de sin embargo, a pesar de que, aunque, aun si, etc. (realizaciones de un conector abstracto SIN EMBARGO, abreviado como SE).

Por otro lado, el EDAP hace suya también la crítica polifónica al postulado de la unicidad del sujeto hablante, según el cual para un enunciado hay un único sujeto, es decir un único individuo que es, a la vez, el responsable de las actividades psicofisiológicas de las que depende la producción del enunciado y el responsable de todo cuanto se comunica en él. Contrariamente a esta visión, el EDAP sostiene que el sujeto hablante es exterior al sentido, por lo que no constituye una variable de análisis y que, en cambio, resulta ineludible distinguir diferentes formas de plasmación de la subjetividad. Entre ellas, resaltamos aquí las figuras del locutor en tanto tal (abreviado L) y del locutor en tanto ser del mundo (abreviado $\lambda$ ). Siguiendo a Ducrot (1984), definimos a L como el responsable de la enunciación según el enunciado, o sea, el locutor considerado únicamente en su compromiso enunciativo. El locutor $\lambda$ es, en cambio, aquel a quien remiten las marcas de primera persona y de quien se habla en el enunciado que contiene dichas 
marcas. Se trata en ambos casos de representaciones discursivas del yo que, en relación dialógica, forman parte del sentido.

Al mismo tiempo, y de acuerdo con Bajtín (1982), el EDAP concibe el enunciado como un eslabón en la cadena discursiva, por lo que los diferentes posicionamientos subjetivos que quedan plasmados argumentativamente en el discurso son analizados como respuestas siempre dialógicas más allá de toda intención retórica o estratégica del hablante. Como afirma Bajtín, toda palabra viva (es decir, la empleada en el discurso) supone una articulación con palabras previas o posteriores, efectivas o no, e inscribe en sí misma una posición subjetiva definida por su relación con la alteridad. Según señala el teórico ruso, la "palabra ajena" es un antecedente inevitable para todo hablante: identidad y alteridad son conceptos interdependientes porque el discurso propio se construye en una relación constitutiva con el discurso ajeno. Y esa relación con el discurso ajeno se vincula con lo que Authier-Revuz (1984) denomina las heterogeneidades enunciativas. Según la autora son dos los tipos de heterogeneidades que "alteran la imagen de un mensaje monódico" (p. 98)2: la constitutiva y la mostrada. La primera da cuenta del hecho de que, a pesar de la pretensión del sujeto de ser fuente autónoma del sentido, el discurso está siempre constituido por otros discursos; la segunda, que perturba la unicidad aparente del hilo del discurso, incorpora la alteridad con o sin señales unívocas de localización. Authier-Revuz (1984) distingue así dos formas de la heterogeneidad mostrada: las marcadas de las no marcadas. En el primer caso, se trata de formas en las que, al quedar localizada y circunscripta la presencia del otro, el yo recupera la ilusión de ser dueño de las palabras (por ejemplo, el discurso referido en estilo directo, las palabras entrecomilladas, las glosas metaenunciativas). Las formas no marcadas, por su parte, son aquellas en las que la presencia del otro aparece sin marcas explícitas (tal es el caso del discurso indirecto libre, la ironía, la alusión, el pastiche, la metáfora, etc.). Se trata, en este último caso, de formas más arriesgadas ya que, como afirma AuthierRevuz (1984), ellas “juegan con la dilución, la disolución del otro en

2 Las traducciones del texto de J. Authier-Revuz (1984) que aquí se incluyen son nuestras. 
uno, de donde este puede salir enfáticamente confirmado, pero donde también puede perderse" (p. 108).

En lo que sigue, y en el marco de esta propuesta de caracterización del sentido en términos de discursos argumentativos evocados de manera dialógico-polifónica, presentamos un análisis de las secciones dispositivas de distintas resoluciones de organismos académicos referidas al empleo del lenguaje inclusivo en sus dependencias. Como adelantamos, intentaremos demostrar que esos fragmentos proponen sistemáticamente una imagen de la enunciación como causada por cierto tipo de discursos argumentativos previos al tiempo que plasman una representación de un sujeto que reflexiona acerca de su propio decir y busca controlar el sentido marcando su discurso con alguna de las formas de la heterogeneidad mostrada. Retomamos así la noción de instrucción causal propuesta por Ducrot (1984), pero leída en clave dialógica (Authier-Revuz, 1984, 1995; Bajtín, 1982). Dicho de otro modo, en el marco del EDAP, argumentaremos que el posicionamiento subjetivo crítico, combativo, militante o transgresor que se manifiesta en este tipo de enunciaciones con marcas de lenguaje inclusivo surge (es decir, está motivado) como respuesta de L, la representación discursiva del responsable de la enunciación, a distintos tipos de (semi) marcos de discurso dialógicos (en adelante $S M D$ ). En otras palabras, diremos que estas enunciaciones se presentan como causadas por un marco (o un semimarco, según el caso) de discurso que articula discursos relativos a la legislación referida a la no discriminación de las personas o a ciertas prácticas censuradas o reprobables con aserciones sobre $\lambda$, la representación discursiva del locutor en tanto ser del mundo, respecto de esos decires.

Permítasenos una última precisión en relación con el concepto de marco de discurso dialógico (abreviado $M D$ ) que propone el EDAP para poder dar cuenta de los aspectos dialógicos del sentido inscriptos en el enunciado. Inspirado en la noción de cadre de discours (Anscombre 1990) $)^{3}$, el concepto de $M D$ se define como un encadenamiento argumentativo que debe ser recuperado como la causa dialógica de

3 Anscombre 1990 utiliza el concepto de cadre de discours para explicar aquellos contenidos semánticos que, mostrados y no afirmados, actúan como una suerte de telón de fondo a la luz del cual debe comprenderse lo dicho (tal es el caso, entre otros, de los adverbios de enunciación y de los contenidos presupuestos). 
la enunciación y al que ella responde con un cierto posicionamiento subjetivo. Hablaremos de $S M D$ si el primer segmento del $M D$ aparece explicitado en el contexto inmediatamente previo. Se trata de una propuesta para analizar y dar cuenta de los efectos de sentido de los enunciados en tanto consideramos que estos se construyen en relación con los otros discursos que habitan en ellos.

\section{Lenguaje inclusivo, (semi)marcos de discurso dialógicos y resoluciones administrativas}

Como bien señala Tosi (2019), el debate respecto del uso del lenguaje inclusivo en la Argentina tomó amplia notoriedad y difusión -tanto en los medios de comunicación tradicionales como en las redes sociales- con ocasión del tratamiento del Proyecto de Ley de Interrupción Voluntaria del Embarazo en la Honorable Cámara de Diputados de la Nación (Argentina, junio de 2018). En ese contexto, en efecto, comenzaron a irrumpir en el discurso público formas con el morfema $e$ como nueva marca de género. Tal fue el caso de les diputades, o aquelles, utilizadas en un reportaje televisivo por la vicepresidenta del Centro de Estudiantes de la Escuela Carlos Pellegrini:

Entonces, creo que les diputades antes que nada van a tener que plantearse algo que es muy central: mañana y el jueves, ¿quieren pasar a la historia como aquelles que dejaron que cientas de mujeres y cientos de cuerpos gestantes sigamos muriendo? ¿o quieren pasar a la historia como quienes realmente la escriben y quienes legalizan el aborto y quienes evitan la muerte de un montón de compañeras y de un montón de mujeres que hoy en día abortan y que tristemente, lo digo como un hecho de una realidad, van a seguir abortando esté o no esté legalizado el aborto (Mira, 2018).

En tanto cuestionadoras del sistema binario, la interpretación de estas formas exigió necesariamente recuperar un $M D$ al que respondía su enunciación y que explicaba el posicionamiento subjetivo que se evidenciaba en ella. En efecto, confrontativa e incisiva, la enunciación con el morfema $e$ en diputades y aquelles aparece como causada por un $M D$ en el que queda establecida una relación en SE entre el discurso normativo y su no acatamiento por parte de la entrevistada (Discurso normativo D SE Neg. acatamiento de D). A la luz de su 
enunciación, ese discurso normativo queda visto como conservador, androcéntrico y patriarcal en tanto evoca argumentaciones en las que el uso del género masculino, a pesar de ser masculino, puede ser considerado como genérico (masculino SE genérico) y en las que la única opción para la humanidad es el binarismo (humanidad PLT binaris$m o$ ). De hecho, inmediatamente después, y ante la intervención del periodista que le cuestiona el empleo de esas formas, la representante estudiantil afirma:

Yo respeto su manera de hablar. Respete usted la mía, la cual es incluyendo tanto géneros de mujer y varón como los géneros que no se interpelan por ninguno de los dos sexos (Mira, 2018).

A continuación, representamos el MD desencadenante del discurso de la estudiante entre corchetes y la respuesta dialógica en itálicas. Graficamos la relación entre ambos mediante el conector dialógico POR LO TANTO.

[Discurso normativo D (masculino SE genérico/ humanidad PLT binarismo) SE Neg. acatamiento de $\lambda$ de D]

\section{POR LO TANTO}

Enunciación confrontativa e incisiva de L que hace ver el discurso normativo como conservador y androcéntrico: la enunciación con el morfema " $e$ " plasma un posicionamiento discursivo transgresor de la norma y combativo de la identidad binaria.

En otras palabras, la relación dialógica entre el discurso normativo no acatado por conservador y androcéntrico y la enunciación de la líder estudiantil puede glosarse como sigue:

[Como, aunque reconoce su existencia, $\lambda$ no acata el discurso normativo conservador según el cual el masculino es genérico, a pesar de ser masculino, y la humanidad, por ser tal, es binaria], la enunciación de L es confrontativa e incisiva mediante el uso del morfema -e.

La aparición de la $e$ y de las otras formas del lenguaje inclusivo (como la x, el*y la @) motivó y aún sigue motivando reacciones diversas que van desde la aceptación de algunas de las formas no sexistas (como los desdoblamientos, las barras o los sustantivos abstractos o colectivos) a la utilización incondicional y militante del morfema $e$ 
como marca reivindicativa de las identidades no binarias ${ }^{4}$; o desde la desestimación por artificioso o innecesario al rechazo virulento, pasando por el escarnio y la ridiculización. Así, por ejemplo, en la entrevista de la que forma parte el fragmento citado, el periodista (Eduardo Feinmann) se indigna frente a estos usos y materializa en su discurso la posición conservadora opuesta. Su intervención, conforme a la normativa lingüística, que identifica género gramatical masculino con interpretación genérica, pone en evidencia su aceptación de la tradición que impone el binarismo y su rechazo al cambio así como a otras identidades de género.

Son "los diputados", que incluye diputados y diputadas. Los diputados.

El mío es castellano. A mí en el colegio me enseñaron a hablar en castellano, Natalia, y es los diputados y las diputadas. En castellano es así.

Esquemáticamente, representamos la respuesta del periodista como sigue:

[Discurso normativo D (masculino SE genérico/ humanidad PLT binarismo) PLT acatamiento de $\lambda$ de D]

\section{POR LO TANTO}

Enunciación conservadora fuertemente asertiva y confrontativa del discurso del no binarismo (de la interlocutora).

En otras palabras, la relación dialógica entre el discurso normativo acatado porque es la ley y la enunciación del periodista puede glosarse esta vez como sigue:

[Como $\lambda$ acata el discurso normativo conservador según el cual el masculino es genérico, a pesar de ser masculino, y la humanidad, por ser tal, es binaria], la enunciación de L es fuertemente asertiva mediante el uso del masculino genérico y por lo tanto confrontativa respecto del discurso de su interlocutora.

4 Como bien señala Tosi (2019), algunos especialistas diferencian el lenguaje no sexis$t a$, que busca la visibilización de la mujer, del lenguaje inclusivo, que objeta el androcentrismo y el binarismo establecido por el género gramatical y que defiende el uso de la $x$, la @ o la $e$ para mostrar la diversidad de géneros. 
Como se constata, y aun cuando los dos segmentos de los $M D$ fundantes de los discursos de ambos interlocutores estén constituidos por los mismos términos (discurso normativo y acatamiento), dichos $M D$ son totalmente distintos. En efecto, mientras que en el primer caso, el discurso de la norma vigente no es acatado, aun cuando se reconoce su existencia, lo que a su vez lo hace ver como conservador, androcéntrico y binario (discurso normativo SE Neg. acatamiento); en el segundo, es precisamente porque tiene esas mismas cualidades, por lo que se lo acata y se lo respeta (discurso normativo PLT acatamiento). En términos de la teoría de los bloques semánticos (Carel y Ducrot, 2005), los $M D$ en cuestión constituyen argumentaciones conversas, es decir, aquellas en las que el conector (PLT o SE) es remplazado por el otro (PLT por SE o SE por PLT) seguido de negación. La ocurrencia de estas argumentaciones conversas pone de manifiesto, al tiempo que explica, la imposibilidad de todo acuerdo entre ambos interlocutores. Se trata, en efecto, de una escena que, lejos de significar un intercambio fructífero, representa un ejemplo más de diálogos estériles que solo profundizan enfrentamientos. Como bien afirma Bolívar (2019), en el debate actual en torno al lenguaje inclusivo, a menudo "se toman posiciones ideológicas difíciles de conciliar en un diálogo que no siempre es cooperativo, sino más bien conflictivo" (p. 357). Y de hecho, el diálogo democrático que "se caracteriza por la oportunidad de participar en una intervención respetuosa y creer que lo que dicen otros tiene valor” (p. 367) no es el escenario más frecuente en los intercambios relativos al uso de las formas específicas de este tipo de lenguaje.

Sin duda, este acontecimiento discursivo motivó gran número de reacciones y comentarios en distintos ámbitos sociales ${ }^{5}$ que explican, entre otras respuestas, la producción de textos por parte de varias universidades, los que incluían la reflexión en cuanto al uso del lenguaje inclusivo, así como una normativa respecto de su aplicación en sus dependencias ${ }^{6}$. Se trata de resoluciones en las que, a diferencia del caso

5 Entre otros, pueden citarse las notas de opinión de Tosi (2018), Ramírez Gelbes (2018), Kolesnicov (2018) o las entrevistas a especialistas publicadas en medios periodísticos: Galperín (2018), Link (2019), Bossi (2020), Moreno (2020) y Kalinovski (2019; 2020). También las conferencias de expertos en el tema registradas en canales de Youtube: Bentivegna (2019); Bonnin (2019); García Negroni (2019b).

6 Otras respuestas provenientes de órganos estatales son la Guía de uso no discriminatorio del lenguaje, de la Honorable Cámara de Diputados de la Nación, la Resolución 
antes citado, parte del marco fundante de la enunciación aparece explicitado en los Vistos y Considerandos, los que se constituyen así en el primer segmento del $S M D$ al que responde la enunciación global. Debe recordarse aquí que estas órdenes, de carácter general, obligatorio y permanente firmadas por las autoridades de un organismo público, tienen una estructura tripartita: en la primera sección -los VISTOSse citan preceptos y normas que sirven de base o de antecedentes para la decisión. Representamos estos discursos (D) mediante el encadenamiento argumentativo: $\mathrm{X}$ CON Y, en el que CON representa cualquiera de los dos conectores, PLT o SE. En tanto primer segmento del SMD fundante, los Vistos, que evocan leyes, principios y otros antecedentes normativos que se presentan como ampliamente aceptados aparecen conectados mediante un PLT con el acatamiento por parte de $\lambda$ respecto de esos discursos o decires. Ahora bien, dado que están explícitos en el texto y que, por lo tanto, constituyen el primer segmento del $S M D$, solo encerramos entre corchetes la aserción sobre $\lambda$ respecto de ellos.

\section{Discursos D ( $\mathrm{x}$ CON Y $\quad$ [PLT acatamiento de $\lambda$ de D]}

En la segunda sección -los CONSIDERANDOS- se exponen, describen y fundamentan las razones y circunstancias que se tuvieron en cuenta. Esta vez, en tanto primeros segmentos de otros $S M D$ fundantes de la enunciación global, los Considerandos, que aluden a diferentes discursos circulantes que actualizan la polémica en curso, quedan articulados con otro tipo de afirmaciones sobre $\lambda$ respecto de ellos: $\lambda$ puede, en efecto, quedar mostrado como estando de acuerdo o no con dichos discursos. En el primer caso, se mantiene la misma argumentación, es decir, la misma interdependencia semántica entre los segmentos $\mathrm{X}$ e $\mathrm{Y}$ articulados por el mismo conector (PLT o $S E$ ); en el segundo, el no acuerdo de $\lambda$ lleva a la argumentación conversa, es decir, a una argumentación en la que los segmentos $\mathrm{X}$ e $\mathrm{Y}$ aparecen conectados por el otro conector seguido de la negación. Dicho de otro modo, si el discurso D está representado por X CON Y, su converso es X CON' Neg. Y y si D está representado por X CON Neg. Y, su converso es X CON’Y.

del Instituto Nacional de Servicios Sociales para Jubilados y Pensionados, PAMI, así como de otras normativas de distintas municipalidades de la provincia de Buenos Aires e incluso del estado provincial. 


\section{Discursos D(X CON Y / X CON Neg. Y)}

[PLT acuerdo de $\lambda$ con D] $\rightarrow$ se mantiene la misma argumentación (X CON Y / X CON Neg. Y)

[SE Neg. acuerdo de $\lambda$ con D] $\rightarrow$ argumentación conversa (X CON' NEG. Y // X CON' Y)

Finalmente, en la parte dispositiva -los RESUELVE-, aparece lo resuelto o dispuesto, por la autoridad competente. En términos del EDAP, esta última sección corresponde a la respuesta dialógica del texto global y en ella queda marcado el posicionamiento de un sujeto que busca controlar, en el marco de la institución que representa, los discursos que pueden circular en ella. Nuevamente graficamos dicha respuesta mediante un POR LO TANTO dialógico

POR LO TANTO

\section{Enunciación resolutiva que busca el control del decir}

Consideremos, a modo de ejemplo, el caso de la resolución de FSOC de la UBA, que reproducimos a continuación.

BUENOS AIRES, O 2 JUL 2019

VISTO, la Convención sobre la eliminación de todas las formas de discriminación contra la mujer, la Convención Americana sobre Derechos Humanos, la Convención Interamericana para prevenir, sancionar y erradicar la violencia contra la mujer, los Principios de Yogyakarta, la Ley de Protección Integral a las Mujeres N 26.485, la Ley de Identidad de Género N² 26743; Y

CONSIDERANDO:

Que en los últimos años se han producido en distintas partes del mundo y en nuestro país importantes avances en materia de derechos de las mujeres, la diversidad sexual y de género.

Que para que la igualdad jurídica se traduzca en igualdad efectiva es necesaria una profunda transformación en las prácticas sociales.

Que resulta necesario que las dinámicas y culturas institucionales acompañen esas transformaciones y fomenten el fortalecimiento de la democracia. 
Que los instrumentos administrativos y de gestión de la Facultad conservan formulaciones y expresiones que no han sido actualizadas contemplando a los sujetos de derechos reconocidos en los últimos años.

Que el lenguaje con el cual nos comunicamos y relacionamos comporta sentidos que reflejan desigualdades entre los géneros, naturalizando la segregación discriminación o exclusión.

Por ello;

\section{EL CONSEJO DIRECTIVO DE LA FACULTAD DE CIENCIAS SOCIALES}

RESUELVE:

ARTÍCULO $1^{\circ}$._ Reconocer el lenguaje inclusivo en cualquiera de sus modalidades como recurso válido en las producciones realizadas por estudiantes de grado y posgrado.

ARTÍCULO $2^{\circ}$._ Encomendar a la Subsecretaría de Políticas de Género que a futuro instrumente acciones de capacitación y difusión a fin de comprometer a la comunidad universitaria a comunicarse con un trato respetuoso de los derechos de las mujeres la diversidad sexual y de género.

ARTÍCULO $3^{\circ}{ }_{-}$. Regístrese y comuníquese a las Secretarías, a la Subsecretaría de Políticas de Género, a las Direcciones de Carrera e Institutos, y a la comunidad académica a través de sus canales institucionales. Cumplido, archívese.

Tal como puede constatarse, los tres artículos que constituyen la sección dispositiva y en los que no aparecen (o se evitan) las formas del masculino genérico (por ejemplo, el sustantivo epiceno estudiantes o los sustantivos colectivos o abstractos comunidad universitaria y comunidad académica) responden, en primer lugar, al SMD conformado por los Vistos que quedan articulados mediante el conector normativo PLT con la adhesión a ellos.

Discursos D (violencia contra mujeres PLT reprobación) // (nuevas identidades PLT no binarismo) [PLT adhesión de $\lambda$ a D]

POR LO TANTO

Enunciación resolutiva que reafirma esos discursos y que busca controlar el decir 
Pero la resolución también responde a los $S M D$ en los que quedan incluidos los Considerandos. En este caso,

Discursos D (avances en materia de derechos SE existencia de prácticas conservadoras y discriminatorias) [PLT adhesión de $\lambda$ a D]

Discursos D (existencia de prácticas discriminatorias PLT necesidad de transformación) [PLT adhesión de $\lambda$ a D]

Discursos D (subsistencia de prácticas discriminatorias en el lenguaje PLT necesidad de transformación) [PLT adhesión de $\lambda$ a D]

\section{POR LO TANTO}

Enunciación resolutiva de reconocimiento y de legitimación del uso del lenguaje inclusivo y de orden para la creación de un área de capacitación como modo de transformación de las prácticas discursivas discriminatorias.

Las resoluciones restantes de nuestro corpus responden al mismo tipo de $S M D$ relativo a los Vistos. En todos los casos, quedan allí incluidas las leyes de no discriminación, de diversidad de género, de no violencia hacia las mujeres, etc. En algunos casos, como en el de FFyL, se agrega la Ley 26.150/2006 de Educación Sexual Integral (ESI) y, en otros, como en el de la UNER, se alude a otros antecedentes normativos procedentes de distintas universidades. Es importante señalar, no obstante, que el orden en el que aparecen las normas que sirven de base o de antecedentes para la decisión resulta significativo. Así, por ejemplo, a diferencia de la resolución de FSoc en la que se alude, en primer lugar, a las leyes y convenciones sobre la discriminación y violencia contra la mujer, en la de la FFyL son las leyes relativas a la diversidad de género y a la ESI las que encabezan los Vistos:

\section{VISTO}

La Ley No 26.743/2012 de Identidad de Género; la Ley 26.150/2006 de Educación Sexual Integral; la Ley 26.485/2009 de Protección integral para prevenir, sancionar y erradicar la violencia contra las mujeres; y la Ley 27.499/2019, “Ley Micaela” de capacitación en género obligatoria a todas las personas que integran los tres poderes del Estado.

Es probable que ello se relacione con el hecho de que, en lugar de la simple evitación del masculino genérico como ocurre en la de FSoc, 
en esta resolución, sea la $x$ la forma de la heterogeneidad mostrada marcada (Authier-Revuz, 1984) que aparece en la producción del propio discurso. De acuerdo con Tosi (en prensa), en tanto huellas de esa heterogeneidad, tanto la $x$, de la que se trata aquí, como la @, el *y la - $e$ ponen en evidencia un comentario del sujeto sobre su propia enunciación. Según la autora, dicho comentario podría glosarse como

x/@/-e/* es lo que conviene/corresponde;

x/@/-e/*a falta de otro recurso;

-o y -a no son válidos, por lo tanto, x/@/-e/*;

x/@/-e/* con valor más amplio o universal;

x/@/-e/*, como dicen los estudios de género, o como recomiendan las guías de lenguaje inclusivo.

Por nuestra parte, agregamos que dichas glosas o comentarios se explican, precisamente, en relación con los $S M D$ que, en cada caso, la enunciación que contiene las formas de la heterogeneidad mostrada exige recuperar como su causa.

Discursos D (identidad de género, ESI, nuevas identidades PLT no binarismo) // (violencia contra mujeres PLT reprobación) [PLT adhesión de $\lambda$ a D]

\section{POR LO TANTO}

Enunciación resolutiva que reafirma esos discursos y que busca controlar el decir

En tanto respuesta dialógica no solo a los Vistos, sino también a los Considerandos, la sección dispositiva de la resolución de FFyL presenta matices de sentido que la diferencian de las otras resoluciones de nuestro corpus. De hecho, en ella, no solo se adopta de manera rigurosa el uso de la $x$ tanto en sustantivos, como en adjetivos y determinantes para referir a los seres sexuados (por ejemplo, interpeladx, representadx, lxs hablantes, juradxs), sino también el de otras formas abstractas que evitan el uso sexista del lenguaje (cf.jefaturas), como se observa en los segmentos que a continuación se incluyen:

una parte de la comunidad universitaria no se siente interpeladx ni representadx por el binarismo de género. 
dichos cambios no se pueden controlar, impedir ni imponer a partir de marcos normativos y regulatorios, sino que son consecuencia del uso lingüístico de lxs hablantes.

Que de ninguna forma la falta de legislación al respecto puede ser motivo de rechazo, sanción o punición por parte de 47 evaluadores, juradxs, jefaturas, etc. y que su uso no puede ser motivo de obligación o coerción.

Asimismo, los Considerandos que en ella aparecen exhiben dos particularidades. En primer lugar, hacemos notar además de la mayor extensión en su desarrollo, la fuerte impronta -en concordancia con los Vistos- de los discursos que legitiman o que reconocen la diversidad de género (y no solo la discriminación o la violencia contra la mujer): a diferencia de la resolución de FSoc o de la de la UNSAM, 7 de los 13 considerandos tematizan esta diversidad o hacen hincapié en ella bajo la forma de la ampliación de derechos. A continuación, reproducimos y resaltamos en itálicas los segmentos más relevantes:

1. Que la Ley de Identidad de Género, en su artículo 1 inciso c, establece que toda persona tiene derecho a ser tratada de acuerdo con su identidad de género, y que una parte de la comunidad universitaria no se siente interpeladx ni representadx por el binarismo de género de nuestra lengua en los casos que refieren a personas.

2. Que el lenguaje inclusivo constituye una intervención política relevante sobre la lengua y que esa intervención es contemporánea de movilizaciones populares, de una multiplicidad heterogénea de militancias del movimiento de mujeres, y de reconocimiento y defensa de la diversidad sexual.

6. Que la Facultad de Filosofía y Letras de la Universidad de Buenos Aires ha acompañado y acompaña estas luchas en sus múltiples manifestaciones.

8. Que este Consejo Directivo comprende la complejidad que tienen los actos administrativos, en especial los documentos dirigidos a otrxs interlocutorxs institucionales, pero es de fundamental importancia que esta Facultad pueda ser receptora de documentos en lenguaje inclusivo, y en la medida de lo posible acompañar la 
transición y generar una tendencia con respecto a esta ampliación de derechos.

9. Que esta Facultad ya ha realizado importantes avances en la ampliación de derechos a la identidad de género y contra la violencia de género, como lo demuestra la conformación de la Comisión No a la Violencia de Género, la implementación del Protocolo UBA, la licencia por violencia de género, la creación de la Cátedra Libre de Estudios Trans, entre otras reivindicaciones.

11. Que el Consejo Superior de la Universidad de Buenos Aires aprobó en su sesión del día 13 de noviembre la implementación de la Ley Micaela, que establece la capacitación obligatoria en la temática de género y violencia de género para todas las personas que se desempeñen en el ámbito de esta universidad,

13. Que es voluntad de este Consejo Directivo incluir y ampliar los derechos a la identidad de género de toda la comunidad universitaria.

Paralelamente, cabe señalar la inclusión argumentada de una reflexión en torno al lenguaje y su carga ideológica y política, el cambio lingüístico y el problema de la inteligibilidad de las prácticas sociales relacionadas con el uso del lenguaje inclusivo.

En otras palabras, y si bien, al igual que en el caso de la de FSoc, el posicionamiento subjetivo que aparece plasmado en la sección dispositiva responde a los $S M D$ que surgen de los Vistos y de los Considerandos, en el caso de FFyL, resulta significativa la presencia de varias negaciones metadiscursivas que refutan las posiciones conservadoras adversas y que muestran un sujeto con un alto grado de polemicidad. Nos permitimos recordar aquí que las negaciones metadiscursivas vehiculizan puntos de vista evidenciales citativos (García Negroni, 2018a, 2019a). En tales casos, estos puntos de vista instan a recuperar un $M D$ del tipo Dicen X SE Neg. acuerdo de $\lambda \operatorname{con} X$, al que la enunciación responde refutando mediante el discurso $D$ con la argumentación conversa, eventualmente seguido de un enunciado de rectificación. A modo de ejemplo, proponemos detenernos en los considerandos (3), (5) y (7) que presentan este tipo de negaciones. En todos los ejemplos, representamos el $M D$ fundante entre corchetes y la respuesta dialógica refutativa en itálicas. 
3. Que las lenguas están en constante proceso de cambio, que las múltiples variedades de nuestra lengua también son producto de esos permanentes cambios, y que dichos cambios no se pueden controlar, impedir ni imponer a partir de marcos normativos y regulatorios, sino que son consecuencia del uso lingüistico de lxs hablantes.

[Discursos normativistas regulatorios $\mathrm{X}$ (marcos normativos y regulatorios PLT Neg. cambio lingüístico) SE Neg. acuerdo de $\lambda \operatorname{con} X]$ POR LO TANTO

ENUNCIACIÓN REFUTATIVA: Discurso D con argumentación conversa (marcos normativos y regulatorios SE cambio lingüístico) + ENUNCIADO DE RECTIFICACIÓN (uso PLT cambio)

5. Que en la medida en que se trata de un fenómeno discursivo cuyo sentido y eficacia pertenecen a las tensiones y disputas por transformar el mundo social, de ninguna manera comporta un riesgo de ininteligibilidad de la lengua.

[Discursos normativistas regulatorios $\mathrm{X}$ (lenguaje inclusivo PLT Neg. inteligibilidad) SE Neg. acuerdo de $\lambda$ con $X]$

POR LO TANTO

ENUNCIACIÓN REFUTATIVA: Discurso D con argumentación conversa (lenguaje inclusivo SE inteligibilidad)

7. Que de ninguna forma la falta de legislación al respecto puede ser motivo de rechazo, sanción o punición por parte de 47 evaluadores, juradxs, jefaturas, etc. y que su uso no puede ser motivo de obligación o coerción.

[Discursos normativistas regulatorios X (falta de legislación PLT reprobación) SE Neg. acuerdo de $\lambda$ con X]

[Discursos inclusivos fundamentalistas X (obligación de no discriminación PLT lenguaje inclusivo obligatorio) SE Neg. acuerdo $\operatorname{de} \lambda \operatorname{con} \mathrm{X}]$

POR LO TANTO

ENUNCIACIÓN REFUTATIVA: Discurso D con argumentación conversa (falta de legislación SE Neg. reprobación / obligación de no discriminación SE Neg. obligación de lenguaje inclusivo) 
Así, mediante estos Discursos D, es decir, mediante estas enunciaciones refutativas en las que se responde con la argumentación conversa a los discursos normativistas regulatorios e incluso a los discursos inclusivos fundamentalistas, queda materializado un posicionamiento subjetivo altamente polémico que legitima, sin prescribir, los usos inclusivos del lenguaje.

En suma, recuperar los $M D$ y los $S M D$ de los que forman parte los Vistos y los Considerandos de esta resolución permite dar cuenta de la causa de la enunciación dispositiva y explicar así los efectos de sentido que en ella quedan plasmados. En efecto, en respuesta dialógica a esos $M D$ y $S M D$, la enunciación muestra un sujeto que, inscripto en una polémica sobre los usos sexistas del lenguaje, busca controlar reflexiva y militantemente su discurso, así como la producción discursiva que se genera en el marco de sus dependencias y más allá de ellas.

VISTOS:

Discursos D (identidad de género, ESI, nuevas identidades PLT no binarismo) // (violencia contra mujeres PLT reprobación) [PLT adhesión de $\lambda$ a D]

\section{CONSIDERANDOS:}

Discursos D (marcos normativos y regulatorios SE cambio lingüístico // uso PLT cambio // lenguaje inclusivo SE inteligibilidad // falta de legislación SE Neg. reprobación // obligación de no discriminación SE no obligación de lenguaje inclusivo) [PLT adhesión de $\lambda$ a D]

\section{POR LO TANTO}

Enunciación resolutiva de reconocimiento y legitimación del lenguaje inclusivo. L marca su enunciación con x / sustantivos abstractos y muestra su posicionamiento discursivo reflexivo y militante sobre la validez del uso del lenguaje inclusivo, promueve esa reflexión e insta al CS de la UBA a reconocer y habilitar el uso de este lenguaje.

Ahora bien, a pesar de las diferencias de sentido señaladas entre las distintas resoluciones, queda claro que tanto en unas como en otras se advierte la presencia de un sujeto que intenta controlar la producción discursiva (propia y ajena). No obstante, como afirma Authier-Revuz (1984), distintos enfoques teóricos han revelado que, ante la preten- 
sión del sujeto de ser fuente autónoma de un sentido, "toda habla está determinada más allá de la voluntad de un sujeto" (p. 99). Muestra de ello son algunas comunicaciones emitidas por la $\mathrm{FFyL}^{7}$ en las que, aun cuando predomina en ellas el uso del lenguaje inclusivo, aparecen otros términos que no lo adoptan. En el ejemplo que sigue, adultes, les invitamos a revisitar juntes (resaltados con itálicas) alternan con viajeros y adultos (resaltados en negrita).

PUBLICADO EL 03.03.2020

Todo marzo en el Museo Etnográfico

viernes 20 de marzo, 18 horas. Celebramos el día de la Narración Oral 2020 con historias de viajes y viajeros

Este año les invitamos a compartir historias, mitos y leyendas sobre viajes y viajeros en las voces de Juan Tapia y Anabelle Castaño. Espectáculo de narración oral a la gorra.

domingo 22 de marzo, 16 horas. Museo regenerado

Visita guiada para adultes. ¿Es posible repensar las colecciones, las exhibiciones y los relatos de un museo desde una mirada de género? Les invitamos a revisitar juntes las salas de este museo a partir de nuevas preguntas.

sábado 28 de marzo, 16 horas. En lo profundo de los Andes. Historias sobre minería

Nueva visita guiada para adultos y adolescentes por distintas salas del Museo sobre la minería andina en tiempos prehispánicos, coloniales y en el último siglo.

La dificultad de "controlar" el propio discurso también puede observarse en tramos de la entrevista a la estudiante Natalia Mira a la que nos hemos referido al inicio de este apartado. Pese a la defensa que la estudiante hace a favor del lenguaje inclusivo y de la alta recurrencia del uso de formas con el morfema $e$, en un tramo de la misma entrevista, irrumpe les padres y las madres. Del mismo modo, en otra entrevista televisiva, una estudiante que pertenece al mismo grupo estudiantil, se autocorrige y dice nosotras, bueno, nosotres, hombres y mujeres.

7 Sección Filo Noticias, disponible en http://filo.uba.ar/ 
En síntesis, los posicionamientos enunciativos críticos, transgresores, combativos, militantes que quedan plasmados en el tipo de discursos aquí analizados (resoluciones universitarias y producciones orales de estudiantes), materializan sujetos con la ilusión renovada de ser dueños de su decir pero que, con frecuencia, mucho más que hablar, son hablados (Authier-Revuz, 1984).

\section{Conclusiones}

El objetivo de este trabajo ha sido sumarnos a la discusión actual acerca de los usos del lenguaje inclusivo. En términos generales, podría decirse que existe un número considerable de voces que adhieren o rechazan estos usos lingüísticos. Por nuestra parte, nuestro interés se ha centrado en el análisis lingüístico-discursivo de una serie de resoluciones universitarias sin afán alguno de establecer juicios que autoricen o nieguen la pertinencia de esos usos. Nuestro propósito ha sido demostrar que el sentido de los discursos no es de naturaleza veritativa, ni depende de las elecciones voluntarias e intencionales de los sujetos. Contrariamente, consideramos que los efectos de sentido se construyen dialógica y argumentativamente en relación con (S)MD que, en el caso particular de las enunciaciones responsivas con lenguaje inclusivo, remiten a prácticas discursivas que toman como objeto el uso mismo del lenguaje y su correlato en las prácticas sociales. Al mismo tiempo, hemos dado cuenta de cómo en esas respuestas dialógicas quedan materializados posicionamientos subjetivos diversos.

En efecto, lejos de ser homogéneos, se trata de sujetos que al regular el uso del lenguaje inclusivo en el marco de sus dependencias adoptan distintas formas de la heterogeneidad mostrada: en algunos casos, se utilizan formas que evitan el masculino genérico y construcciones en las que la presencia del otro aparece sin marcas explícitas (como ocurre en las resoluciones de FSoc y de UNSAM); en otros, se opta por formas de la heterogeneidad mostrada marcada. Tal el caso de la $x$ a la que adhiere FFyL o de la $e$ en la propia producción en el caso de la UNER (universidad en la que también se autorizan la $x$ u "otras formas para evitar usar el género masculino al referirse a todos los géneros"). La utilización de una u otra opción no es la única diferencia en el proceso de constitución de la subjetividad en estas resoluciones. 
Dicho proceso debe relacionarse también con el grado de polemicidad respecto de los discursos relativos tanto a la discriminación de las personas, como a ciertas prácticas censurables o reprobables. Y también con la mayor o menor puesta en foco de las diversidades identitarias: en algunos casos, y aunque se hace mención de la diversidad sexual y de género, es cuestión sobre todo de la visibilización de la mujer en las prácticas discursivas; en otros, el foco está puesto también en el reconocimiento de otras identidades históricamente silenciadas (tal el caso de FFyL y de la UNER). Finalmente, si bien en todas las resoluciones se adhiere y promueve el uso del lenguaje inclusivo, en la de FFyL ese uso no reviste carácter obligatorio: como bien es sabido -y todas las resoluciones lo ponen de manifiesto-, los modos de hablar no son susceptibles de imposición y "el uso del lenguaje no se decreta" (Bolívar, 2019, p. 362).

Más allá de los pronósticos y predicciones que algunos podrán hacer acerca del futuro, el desafío al que nos enfrenta la legitimación/ autorización/incorporación del lenguaje inclusivo es cómo dar cuenta de la diversidad de identidades que se hacen por cierto visibles. Como bien afirma Maffia (2019, p. 1), “[c]ualquiera sea el modo en que el lenguaje nos refiera, siempre lo hará bajo la forma de condiciones universales que pueden ser o no cumplidas por nosotrxs, pero que nunca agotarán la descripción lo suficiente como para alcanzarnos en toda nuestra complejidad".

\section{Referencias bibliográficas}

Anscombre, J. -C. (1990). Thème, espace discursif et représentations événementielles. En J.-C. Anscombre \& G. Zaccharia (Eds.), Fonctionnalisme et pragmatique: à propos de la notion de thème (pp. 43-150). Milan: Unicopli.

Anscombre, J. -C. (1995). Théorie des Topoi. Paris: Kimé.

Anscombre, J. -C. \& Ducrot, O. (1983). L'argumentation dans la langue. Lieja: Mardaga.

Authier-Revuz, J. (1984). Hétérogénéité(s) énonciative(s). Langages, 19(73), 98-111. https://doi.org/10.3406/lgge.1984.1167

Authier-Revuz, J. (1995). Ces mots qui ne vont pas de soi. Boucles réflexives et non-coïncidences du dire. Paris: Larousse.

Bajtín, M. (1982). Estética de la creación verbal. México, D.F.: Siglo XXI. 
Bentivegna, D. (2019). Diego Bentivegna habla sobre lenguaje inclusivo. Recuperado de https://www.youtube.com/watch?v=tM6JG$\mathrm{y}-5 \mathrm{SE}$.

Bolívar, A. (2019). Una introducción al análisis crítico del 'lenguaje inclusivo'. Literatura y Lingüística, 40, 355-375. https://doi. org/10.29344/0717621x.40.2071

Bonnin, J. E. (2019). Lenguaje inclusivo: ¿Cómo se transforma el idioma? Recuperado de https://www.youtube.com/watch?v=8zuPySZ0hY

Bossi, E. (2020). No molesta el lenguaje inclusivo, lo que molesta es el feminismo. Entrevista de C. Amador. Recuperado de https:// www.eltribuno.com/jujuy/nota/2020-1-26-0-0-0--no-molesta-el-lenguaje-inclusivo-lo-que-molesta-es-el-feminismo.

Carel, M. y Ducrot, O. (2005). La semántica argumentativa. Una introducción a la teoría de los bloques semánticos. Buenos Aires: Colihue.

Ducrot, O. (1984). Le dire et le dit. Paris: Minuit.

Ducrot, O. (2004). Sentido y argumentación. En E. Arnoux y M. M. García Negroni (Eds.), Homenaje a Oswald Ducrot (pp. 359-370). Buenos Aires: Eudeba.

Galperín, K. (2018). La sobreactuación contra el lenguaje inclusivo es esperable porque es una disputa entre generaciones. Entrevista de M. Fernández. Infobae. Recuperado de https:// www.infobae.com/cultura/2018/08/04/karina-galperin-lasobreactuacion-contra-el-lenguaje-inclusivo-es-esperableporque-es-una-disputa-entre-generaciones/

García Negroni, M. M. (2016). Polifonía, evidencialidad citativa y tiempos verbales. Acerca de los usos citativos del futuro morfológico y del futuro perifrástico. En R. González Ruiz, D. Izquierdo Alegría, y O. Loureda Lamas (Eds.), La evidencialidad en español: teoría y descripción (pp. 279-302). Madrid: Iberoamericana.

García Negroni, M. M. (2018a). Argumentación y puntos de vista evidenciales citativos: acerca de la negación metadiscursiva en el discurso político. Oralia, 21(2), 223-242.

García Negroni, M. M. (2018b). Argumentación y puntos de vista evidenciales: acerca del condicional citativo en el discurso periodístico y en el discurso científico. Boletín de Lingüistica, 
30(49-50), 86-109. Recuperado de https://dialnet.unirioja. es/servlet/articulo?codigo $=7021538$ \&orden $=0$ \&info=link

García Negroni, M. M. (2019a). El enfoque dialógico de la argumentación y la polifonía, puntos de vista evidenciales y puntos de vista alusivos. RILCE: Revista de Filología Hispánica, 35(2), 521-549. https://doi.org/10.15581/008.35.2.521-49

García Negroni, M. M. (junio, 2019b). Acerca del lenguaje inclusivo. Recuperado de https://www.youtube.com/ watch? $\mathrm{v}=\mathrm{ZnKtp} 84 \mathrm{~S} 5 \mathrm{X} 0$

García Negroni, M. M. y Libenson, M. (2020). La evidencialidad desde el enfoque dialógico de la argumentación y de la polifonía. Un estudio contrastivo de los empleos inferencial y citativo del marcador evidencial así que. En Ó. Loureda Lamas, M. Rudka, y G. Parodi (Eds.), Marcadores del discurso y lingüística contrastiva en las lenguas románicas (pp. 41-62). Madrid: Iberoamericana.

García Negroni, M. M. y Libenson, M. (en prensa). Aportes del enfoque dialógico de la argumentación y de la polifonía al estudio del significado evidencial: ¿ Y (tono circunflejo)...X? y ¿Así que $\mathrm{X}$ ? en contraste. En C. Fuentes Rodríguez, A. Messias Nogueira, y M. Martí (Coords.), Nuevas perspectivas sobre los marcadores del discurso: descripción y contraste. Sevilla: Editorial de la Universidad de Sevilla.

Kalinowski, S. (25 de abril de 2019). Lenguaje inclusivo. Entrevista de I. Goano y M. Coronel. Hoy nos toca a las Diez [Transmisión televisiva]. Buenos Aires: Canal de la Ciudad. Recuperado de https://www.youtube.com/watch?v=hFSzr2iRyeo

Kalinowski, S. (13 de enero de 2020). Santiago Kalinowski: "Prohibir la 'e' es persecución política". Entrevista de S. Santoro. Página/12. Recuperado de https://www.paginal2.com. ar/241461-santiago-kalinowski-prohibir-la-e-es-persecucion-politica

Kolesnicov, P. (2018). ¿Chicos, chicas o "chiques"? El gran debate del lenguaje inclusivo. Recuperado de https://www.clarin.com/ opinion/vienen-chiques_0_HyS7IzURz.html.

Link, D. (8 de abril de 2019). Las políticas lingüísticas permiten hacerse una pregunta sobre la propia soberanía. Entrevista de Medionegro. La Tinta. Recuperado de https://latinta.com. ar/2019/04/politicas-linguisticas-pregunta-soberania/. 
Maffía, D. (s/f). Lo que no tiene nombre. Recuperado de http://dianamaffia.com.ar/archivos/Lo-que-no-tiene-nombre.pdf.

Martínez, A. (2019). Disidencias en la conformación de la gramática: el lenguaje inclusivo. Heterotopías, 2(4), 1-16. Recuperado de https://revistas.unc.edu.ar/index.php/heterotopias/article/ view/27331

Mira, N. (12 de junio de 2018). Entrevista de E. Feinmann. El noticiero A24 [Transmisión televisiva]. Buenos Aires: A24. Recuperado de https://www.youtube.com/watch?v=W53r_9OpAVM

Moreno, M. (9 de febrero de 2020). Lenguaje inclusivo. Página/12. Recuperado de https://www.paginal2.com.ar/246506-lenguaje inclusivo?fbclid=IwAR33uJwODvxEsRVbbvRbazPtBPPQr zzSlT8U1wkw5guh0r2S-0D6ZjBa45Q

Ramírez Gelbes, S. (2018). Les invito a todes. Recuperado de https:// www.telam.com.ar/notas/201810/297744-les-invito-a-todes--por-silvia-ramirez-gelbes-.html.

Salerno, P. (2019). Lenguaje, género y los límites de la desigualdad. Tábano, 15, 109-115. Recuperado de https://repositorio.uca. edu.ar/handle/123456789/9424

Sarlo, B. y Kalinowski, S. (2019). La lengua en disputa. Un debate sobre el lenguaje inclusivo. Buenos Aires: Godot.

Tosi, C. (2018). Nuestro idioma en el banquillo por machista. Revista N. Recuperado de https://www.clarin.com/revista-enie/ideas/ idioma-banquillo-machista_0_Skx_yuxuX.html

Tosi, C. (2019). Marcas discursivas de la diversidad. Acerca del lenguaje no sexista y la educación lingüística: aproximaciones al caso argentino. ÁLabe, 20. http://dx.doi.org/10.15645/Alabe2019.20.11

Tosi, C. (en prensa). ¿Hojas de estilo para el lenguaje inclusivo? Un análisis de las prácticas de corrección de estilo en el ámbito editorial. Exlibris. 